\title{
ILI and SARI Surveillance along the California \& Arizona Borders with Mexico, 2011-12
}

\author{
Pete Kammerer*, Gary Brice, Anthony Hawksworth and Chris Myers \\ Operational Infectious Diseases, NHRC, San Diego, CA, USA
}

\section{Objective}

To identify the pathogens responsible for influenza-like illness (ILI) and severe acute respiratory illness (SARI) along the U.S.-Mexico border region in San Diego and Imperial Counties, CA and Pima County, AZ.

\section{Introduction}

National borders do not prevent the transmission of pathogens and associated vectors among border populations. The Naval Health Research Center (NHRC) has collaborated with the Mexican Secretariat of Health, the U. S. Department of State's Biosecurity Engagement Program (BEP) and the U. S. Centers for Disease Control and Prevention $(\mathrm{CDC})$ in concert with local health officials to conduct ILI surveillance (since 2004) and SARI surveillance (since 2009) in the border region.

\section{Methods}

Respiratory swabs were collected from patients with ILI (fever $\geq$ $100 \mathrm{~F}$, and sore throat or cough) or SARI ( $\geq 5$ y.o.: ILI with hospital admission; $<5$ y.o.: clinical suspicion of pneumonia with hospital admission) and stored at -70C. Specimens were tested with molecular techniques, viral and bacterial culture.

\section{Results}

NHRC received and tested 295 ILI specimens collected from four surveillance sites in 2011-12. Demographics: $53 \%$ female, $47 \%$ male; $36 \% 0-4$ yrs old, $50 \%$ 5-24 yrs old, $8 \% 25-49$ yrs old, $4 \% 50-64$ yrs old, $2 \%>64$ yrs old. Pathogens identified included influenza A
$(15 \%)$; rhinovirus $(8 \%)$; respiratory syncytial virus (RSV) $(7 \%)$; adenovirus (6\%); influenza B (4\%) and parainfluenza virus (PIV) 1; (4\%). 335 SARI specimens were collected from 6 sites. Demographics: $52 \%$ female, $48 \%$ male; $41 \% 0-4$ yrs old; $9 \% 5-24$ yrs old, $12 \%$ 25-49 yrs old, $11 \% 50-64$ yrs old, $28 \%>64$ yrs old. Pathogens identified included RSV (17\%); rhinovirus (10\%); influenza A $(9 \%)$; adenovirus (6\%); influenza B (2\%) and PIV 1 (1\%).

\section{Conclusions}

In 2011-12, our surveillance identified a difference in the proportion of respiratory pathogens affecting outpatients and inpatients. Influenza A was isolated more frequently in outpatients, whereas RSV was more frequent in hospitalized patients. We also noted an increased proportion of specimens from the 50-64 yr old and the $>64$ yr old age groups in the SARI surveillance, whereas $86 \%$ of the ILI specimens are from patients 24 yrs old or less. Additional benefits of this collaborative surveillance have been the cooperation, joint training and communication between the participating entities. These preestablished lines of communication are invaluable during a public health emergency, which was demonstrated during the recent influenza pandemic.

\section{Keywords}

influenza; ILI; respiratory syncytial virus; US-Mexico border; SARI

\section{*Pete Kammerer}

E-mail: peter.kammerer@med.navy.mil 